\title{
GROUP GENERATED BY HALF TRANSVECTIONS
}

\author{
TAKASHI TSUBOI \\ Dedicated to Professor Tadayoshi Mizutani on his sixtieth birthday
}

\begin{abstract}
Consider the group $S L(2 ; \mathbf{Z})$ acting on the circle consisting of rays from the origin in $\mathbf{R}^{2}$. The action of parabolic elements or transvections $X \in S L(2 ; \mathbf{Z})(\operatorname{Tr} X=2)$ have 2 fixed points on the circle. A half transvection is the restriction of the action of a parabolic element to one of the invariant arcs extended by the identity on the other arc. We show that the group $G$ generated by half transvections is isomorphic to the Higman-Thompson group $T$, which is a finitely presented infinite simple group. A finite presentation of the group $T$ has been known, however, we explain the geometric way to obtain a finite presentation of the group $T$ by the Bass-Serre-Haefliger theory. We also give a finite presentation of the group $T$ by the generators which are half transvections.
\end{abstract}

\section{Introduction}

This paper concerns the following natural question posed by A'Campo to the author. Consider the group $S L(2 ; \mathbf{Z})$ acting on the circle consisting of rays from the origin in $\mathbf{R}^{2}$. Parabolic elements $X \in S L(2 ; \mathbf{Z})(\operatorname{Tr} X=2)$ have 2 fixed points on the circle. These are called transvections. A half transvection is the restriction of the action of a parabolic element to one of the invariant arcs extended by the identity on the other arc. A'Campo asked the nature of the group $G$ generated by the half transvections. This paper shows that the group $G$ is isomorphic to the Higman-Thompson group $T$, which is a finitely presented infinite simple group.

The Higman-Thompson groups are studied by many people. We refer the reader to an excellent reference [4] for the Higman-Thompson group $T$ as well as $F$ which we also need.

1991 Mathematics Subject Classification. Primary 20F32; Secondary 20F05, 20E32, 20F38, 20H10, 57S25, 57S30, 57M07.

Key words and phrases. half transvections, piecewise linear homeomorphisms, the HigmanThompson groups, piecewise projective diffeomorphisms.

The author is supported by Monbusho Zaigai Kenkyuin, Ministry of Education, Science, Sports and Culture, Japan.

Received October 14, 2004; revised January 26, 2005. 
Let $\mathbf{Z}[1 / 2]$ denote the ring of dyadic numbers $\left\{p / 2^{n} ; p \in \mathbf{Z}, n \in \mathbf{Z}_{\geq 0}\right\} \subset \mathbf{R}$. The group $T$ can be defined to be the group of the orientation preserving piecewise linear homeomorphisms $f$ of the circle $\mathbf{R} / \mathbf{Z}$ such that $f$ maps the set of dyadic numbers $\mathbf{Z}[1 / 2] / \mathbf{Z}$ to itself, the nondifferentiable points of $f$ are contained in $\mathbf{Z}[1 / 2] / \mathbf{Z}$, and the slopes of $f$ are powers of 2 .

This group $T$ is isomorphic to the group of orientation preserving automorphisms of the infinity of the infinite trivalent tree $\mathscr{T}$ ([7], [4] where this fact is attributed to Thurston). It is well-known that the group of the orientation preserving automorphisms of the tree $\mathscr{T}$ is isomorphic to $\operatorname{PSL}(2 ; \mathbf{Z})$. Then the group of piecewise $\operatorname{PSL}(2 ; \mathbf{Z}), C^{1}$-diffeomorphisms of the circle at infinity is isomorphic to the group $T$.

To show the isomorphism between the group $G$ generated by half transvections and the group $T$, it is enough to know about above equivalent definitions of the group $T$ and the generators of the group $T$. This is shown in $\S 2$ after a review of the Higman-Thompson groups $T$ and $F$.

The group $F$ is the group of the orientation preserving piecewise linear homeomorphisms $f$ of the interval $[0,1]$ such that the nondifferentiable points of $f$ are contained in $\mathbf{Z}[1 / 2] / \mathbf{Z}$ and the slopes of $f$ are powers of 2 (hence $f$ maps the set of dyadic numbers $\mathbf{Z}[1 / 2] \cap[0,1]$ to itself).

The commutator subgroup $[F, F]$ of the group $F$ coincides with the subgroup of $F$ consisting of elements which are the identity on a neighborhood of $\{0,1\}$. It is easy to show that $[F, F]$ is perfect $([F, F]=[[F, F],[F, F]])$ and this implies that $[F, F]$ is simple $([5],[1])$. Since the group $T$ has fragmentation property, the group $T$ is also a simple group ([5], [1]).

Thus the simplicity of the group $T$ has a geometric proof. We may look for a geometric way to find a finite presentation of the group $T$.

The group $F$ is finitely presented and a finite presentation of $F$ can be obtained by looking at the action of the group $F$ on a certain complex ([2], [3]). For the group $T$, we look at the action of $T$ on the complex consisting of triangle with vertices in $\mathbf{Z}[1 / 2] / \mathbf{Z}$ in $\S 3$. Then by the Bass-Serre-Haefliger theory ([8]), we obtain a finite presentation of the group $T . \quad \$ 3$ also contains a brief review of the Bass-Serre-Haefliger theory.

Since half transvections are also natural generators for the group $T$, we expect to have a simple finite presentation with respect to half transvections. In $\S 4$, we give the presentation of the group $T$ by the generators which are half transvections.

A part of this paper was presented in a lecture at Encounter with Mathematics, at Chuo University, Tokyo, in October 1998. The author thanks Vlad Sergiescu who pointed out several recent references on the Higman-Thompson groups to him. The author also thanks Yakov Eliashberg for his warm hospitality during his stay at Stanford University in 1999, where this paper is written.

\section{The Higman-Thompson groups}

In this section we review the Higman-Thompson groups $F$ and $T$. An excellent reference is the paper [4] by Cannon, Floyd and Parry. These groups 
$F$ and $T$ are represented as groups of piecewise linear homeomorphisms of the interval or of the circle. We can write down a piecewise linear homeomorphism $f$ of $\left[a_{0}, a_{k}\right]$ to itself as follows.

$$
f=P L\left(\begin{array}{c}
a_{0}, \ldots, a_{k} \\
b_{0}, \ldots, b_{k}
\end{array}\right)
$$

where $a_{0}<\cdots<a_{k}, b_{0}<\cdots<b_{k}, a_{0}=b_{0}$, and $a_{k}=b_{k}$. This represents the piecewise linear homeomorphism $f$ of $\left[a_{0}, a_{k}\right]$ such that

$$
f(x)=\frac{b_{i}-b_{i-1}}{a_{i}-a_{i-1}}\left(x-a_{i-1}\right)+b_{i-1} \quad \text { on the interval }\left[a_{i-1}, a_{i}\right] .
$$

This can be described by the rectangle diagram [4]. We prefer to drawing the map being from the right side to the left side of the rectangle as in Figure 2.1.

The Higman-Thompson group $F$ is the group of piecewise linear homeomorphisms $f$ of the interval $[0,1]$ such that the nondifferentiable points of $f$ are
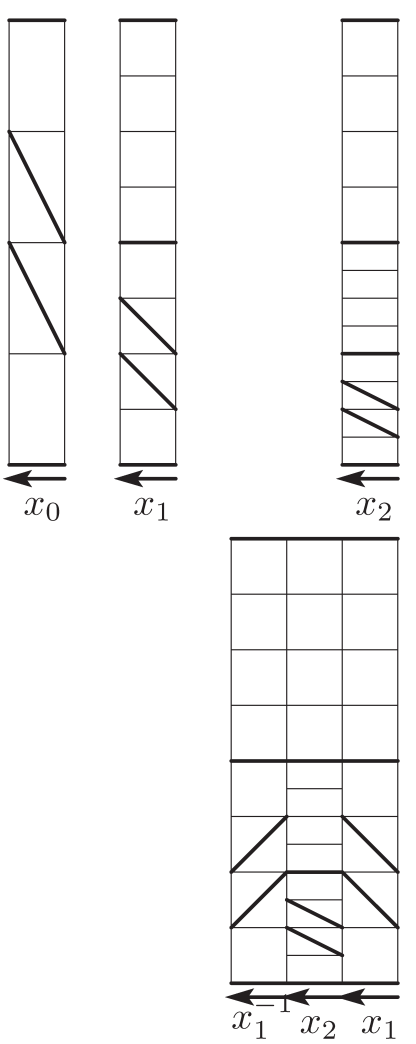
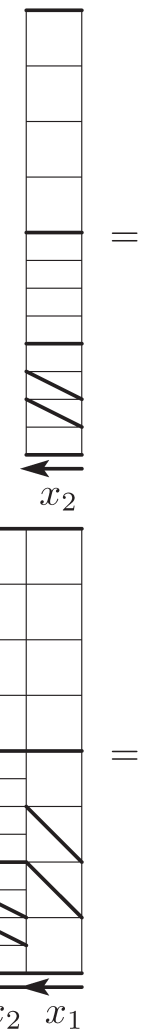
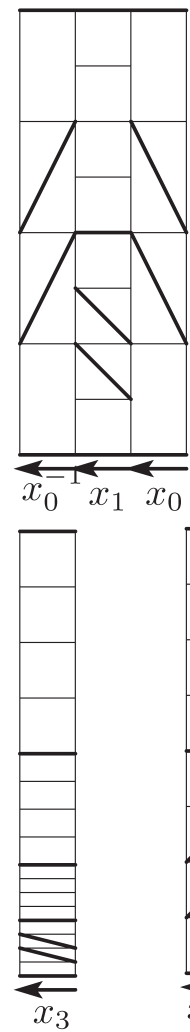
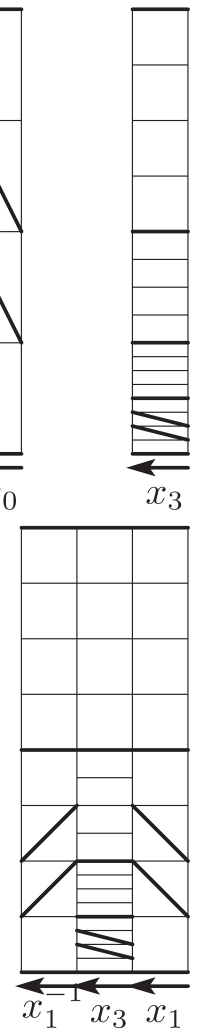

$=$

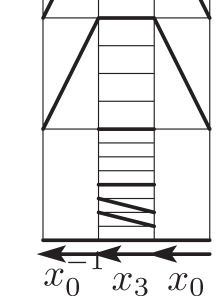

FIGURE 2.1 
contained in $\mathbf{Z}[1 / 2]$ and the slopes of $f$ are contained in $\left\{2^{n} ; n \in \mathbf{Z}\right\}$. Then an element $f$ of the group $F$ maps the set of dyadic numbers $[0,1] \cap \mathbf{Z}[1 / 2]$ to itself.

It is known that the Higman-Thompson group $F$ is generated by the following piecewise linear homeomorphisms $x_{0}$ and $x_{1}$ ([2], [3], [4]);

$$
\begin{aligned}
& x_{0}=P L\left(\begin{array}{llll}
0, & 1 / 4, & 1 / 2, & 1 \\
0, & 1 / 2, & 3 / 4, & 1
\end{array}\right), \\
& x_{1}=P L\left(\begin{array}{lllll}
0, & 1 / 8, & 1 / 4, & 1 / 2, & 1 \\
0, & 1 / 4, & 3 / 8, & 1 / 2, & 1
\end{array}\right) .
\end{aligned}
$$

The rectangle diagrams of $x_{0}$ and $x_{1}$ are shown in Figure 2.1.

A presentation of the group $F$ is given as follows ([2], [3], [4]). This presentation can be obtained by looking at the action of the group $F$ on a certain complex ([2], [3]).

$$
F=\left\langle x_{0}, x_{1}: x_{2}=x_{0}^{-1} x_{1} x_{0}, x_{3}=x_{0}^{-2} x_{1} x_{0}^{2}, x_{1}^{-1} x_{2} x_{1}=x_{3}, x_{1}^{-1} x_{3} x_{1}=x_{0}^{-1} x_{3} x_{0}\right\rangle .
$$

The group $F$ is a group of 2 generators and 2 relations. Figure 2.1 shows that the piecewise linear homeomorphisms $x_{0}$ and $x_{1}$ satisfy the above relations.

The Higman-Thompson group $T$ is defined to be the group of the orientation preserving piecewise linear homeomorphisms $f$ of the circle $\mathbf{R} / \mathbf{Z}$ such that $f$ maps the dyadic numbers $\mathbf{Z}[1 / 2] / \mathbf{Z}$ to itself, the nondifferentiable points of $f$ are contained in $\mathbf{Z}[1 / 2] / \mathbf{Z}$, and the slopes are contained in $\left\{2^{n} ; n \in \mathbf{Z}\right\}$.

A finite presentation of the group $T$ is given in [4]. We explain the way to obtain a finite presentation of the group $T$ by the Bass-Serre-Haefliger theory [8] in $\S 3$.

Now we look at the group $G$ generated by half transvections and show that $G \cong T$.

THEOREM 2.1. Let $G$ be the group of the $C^{1}$ diffeomorphisms of the circle which is generated by half transvections, where a half transvection is the restriction

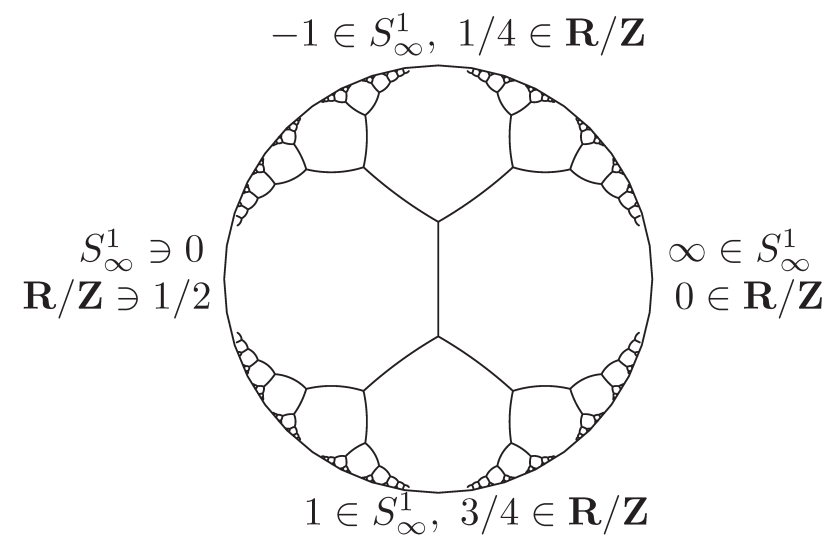

FIGURE 2.2 
of the action on the set of rays of a parabolic element $X \in S L(2 ; \mathbf{Z})$ to one of the invariant arcs extended by the identity on the other arc. Then the group $G$ is isomorphic to the Higman-Thompson group $T$.

In order to look at the relationship between the groups $G$ and $T$, it is better to consider $T$ as the group of orientation preserving automorphisms of the infinity of the infinite trivalent tree $\mathscr{T}([7],[4])$. It is well-known that the group of the orientation preserving automorphisms of the tree $\mathscr{T}$ is $\operatorname{PSL}(2 ; \mathbf{Z})$. Then the group of piecewise $\operatorname{PSL}(2 ; \mathbf{Z}), C^{1}$-diffeomorphisms of the circle at infinity is isomorphic to the group $T$ ([7], [4]).

Now the double covering group $\operatorname{SL}(2 ; \mathbf{Z})$ of $\operatorname{PSL}(2 ; \mathbf{Z})$ acts on the double covering space $\hat{S}_{\infty}^{1}$ of the circle $S_{\infty}^{1}$ at infinity of the hyperbolic plane. For $\hat{S}_{\infty}^{1}$, we consider the double cover of the infinity of the tree $\mathscr{T}$. Since this space is isomorphic to the infinity of the original tree, $S L(2 ; \mathbf{R})$ can be written as a subgroup of $T$.

Remark 2.2. In the same way, the $k$-fold covering group of $\operatorname{PSL}(2 ; \mathbf{R})$ is contained in the group $T$.

It is well-known that $S L(2 ; \mathbf{Z})$ is generated by $\left(\begin{array}{ll}1 & 1 \\ 0 & 1\end{array}\right)$ and $\left(\begin{array}{cc}1 & 0 \\ -1 & 1\end{array}\right)$. As an element of $\operatorname{PSL}(2 ; \mathbf{Z})$, the matrix $\left(\begin{array}{ll}1 & 1 \\ 0 & 1\end{array}\right)$ acts on the tree as a translation fixing $\infty \in S_{\infty}^{1}$ this corresponds to the element $x_{0}$ of the group $F \subset T$. The matrix $\left(\begin{array}{ll}1 & 1 \\ 0 & 1\end{array}\right) \in S L(2 ; \mathbf{R})$ is acting the double cover of the infinity of the tree $\mathscr{T}$, and we see that the action corresponds to the element $x_{1} y_{1}$ in the group $T$, where $y_{1}=x_{0}^{2} x_{1}^{-1} x_{0}^{-1}$. The set of rays is considered with clockwise orientation here, because $x / y$ is considered as an element of the circle at infinity of the hyperbolic plane.

In a similar way, $\left(\begin{array}{cc}1 & 0 \\ -1 & 1\end{array}\right) \in S L(2 ; \mathbf{R})$ corresponds to the element $u_{1} v_{1}$ in the group $T$, where the piecewise linear homeomorphisms $u_{1}$ and $v_{1}$ are drawn in Figure 2.3.

Now the transvections $x_{1} y_{1}, u_{1} v_{1}$ are the products of the half transvections $x_{1}, y_{1}, u_{1}, v_{1}$. We are considering the group $G$ generated by $x_{1}, y_{1}, u_{1}, v_{1}$. Since $x_{1}, y_{1}, u_{1}, v_{1}$ are elements of the group $T$, we see that the group $G$ is a subgroup of $T$. Note that the group $G$ is precisely the group of piecewise $S L(2 ; \mathbf{Z}), C^{1}$-diffeomorphisms of the circle.

Remark 2.3. The group $T$ acts on the dual tesselation of the infinite trivalent tree. This is studied by Penner and others ([11], [12], [9], [10]).

We are going to show that $G=T$. Since the group $G$ is a subgroup of $T$, it is just necessary to show that generators of the group $T$ are written by $x_{1}, y_{1}$, $u_{1}, v_{1}$. 

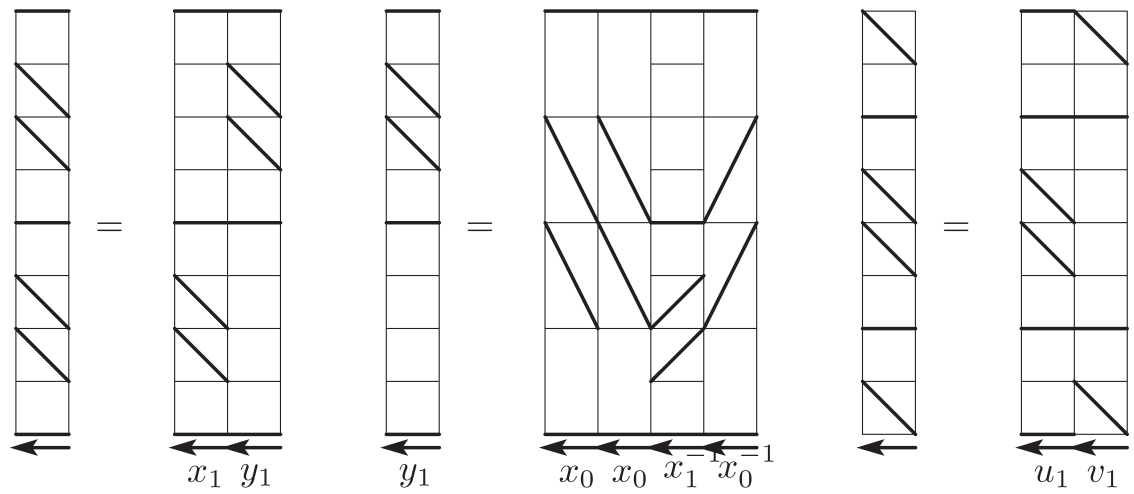

FIGURE 2.3

Proof of Theorem 2.1. The group $T$ is known to be generated by $x_{0}, x_{1}$ and $\omega$, where $\omega$ is represented as a piecewise linear homeomorphism of $\mathbf{R} / \mathbf{Z}$ such that $\omega([0,1 / 4])=[1 / 4,1 / 2], \omega([1 / 4,1 / 2])=[1 / 2,1]$, and $\omega([1 / 2,1])=[0,1 / 4]([4]$, see also §3). (As a piecewise $\operatorname{PSL}(2 ; \mathbf{Z}), C^{1}$-diffeomorphism, $\omega$ is represented by $\left(\begin{array}{cc}1 & 1 \\ -1 & 0\end{array}\right) \in \operatorname{PSL}(2 ; \mathbf{Z})$.)

Now $x_{0}$ and $\omega$ are written as follows (see Figure 2.4).

$$
\begin{aligned}
x_{0} & =y_{1} u_{1} x_{1}, \\
\omega & =x_{1} v_{1} y_{1} y_{1} u_{1} x_{1} .
\end{aligned}
$$

Thus we showed $G=T$.
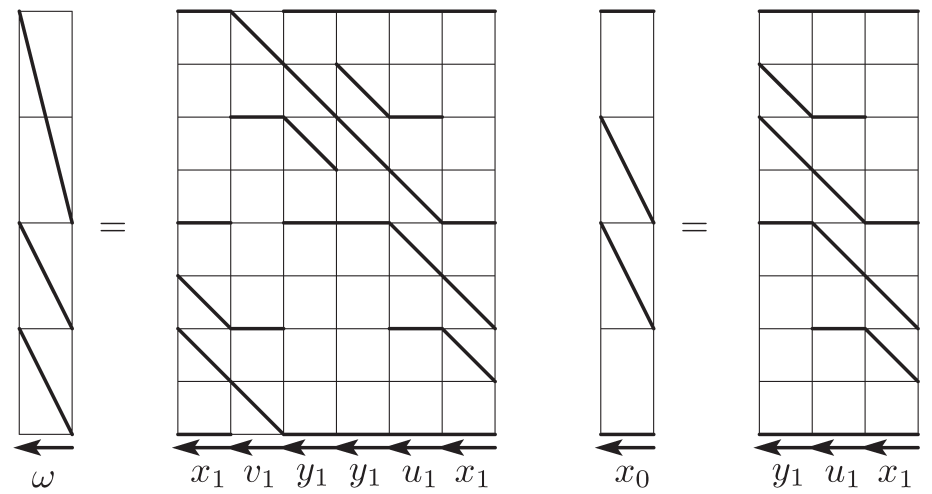

FIGURE 2.4 


\section{A finite presentation of the group $T$}

In this section we explain a method by Haefliger [8] to give a finite presentation of the group $T$ and carry it out.

We consider a simply connected simplicial 2-complex $\tilde{X}$ where the group $T$ acts without inversion. Then the quotient space $X=\tilde{X} / T$ has the structure of a complex of groups. Haefliger showed the following theorem for the finite presentation of the group $T([8])$.

THEOREM 3.1 (Finiteness Theorem [8]). Let $\tilde{X}$ be a simply connected simplicial 2-complex where the group $T$ acts without inversion. If $X=\tilde{X} / T$ is finite complex and the isotropy groups associated to triangles, edges and vertices are finitely presented, then $T$ is finitely presented. Moreover there is an algorithm to give a presentation.

Let $\tilde{Y}$ be the 2-dimensional simplicial complex of the triangles with vertices in the dyadic numbers $\mathbf{Z}[1 / 2] / \mathbf{Z}$. More precisely, let $C_{n}$ be the set of sets of $n$ distinct points of the dyadic numbers $\mathbf{Z}[1 / 2] / \mathbf{Z}$. We have the face operators $C_{n+1} \rightarrow C_{n}$ and its geometric realization is an infinite dimensional simplex with vertices $\mathbf{Z}[1 / 2] / \mathbf{Z}$. Let $\tilde{Y}$ be its 2 -skeleton. Then $\tilde{Y}$ is simply connected as the 2-skeleton of a contractible space.

Since the group $T$ contains the rotation by any number in $\mathbf{Z}[1 / 2] / \mathbf{Z}$, the group $T$ acts transitively on $\mathbf{Z}[1 / 2] / \mathbf{Z}$. The isotropy group of a point is isomorphic to the group $F$. Since for any point $t$ in $(0,1) \cap \mathbf{Z}[1 / 2],[0, t]$ and $[t, 1]$ can be subdivided into a union of intervals of length of powers of $1 / 2$, the group $F$ acts transitively on $(0,1) \cap \mathbf{Z}[1 / 2]$. Hence the group $T$ is doubly transitive on $\mathbf{Z}[1 / 2] / \mathbf{Z}$. Then by the same reasoning, the group $T$ acts transitively on the sets of 3 distinct points of $\mathbf{Z}[1 / 2] / \mathbf{Z}$.

Now we look at the simplicial action of the group $T$ on the simplicial complex $\tilde{Y}$. There are elements of the group $T$ which fix an edge and the restrictions of their actions to the edge are not the identity but the inversions. There are also elements of the group $T$ which fix a triangle and the restrictions of their actions to the triangle are not the identity but the simplicial rotation of order 3.

A simplicial action of a group is said to be without inversion if an element of the group fixes a simplex then the restriction of its action to the simplex is the identity.

Since the action of the group $T$ on the simplicial complex $\tilde{Y}$ has inversions, we look at the action of the group $T$ on the barycentric subdivision $\tilde{X}=\tilde{Y}^{\prime}$ of $\tilde{Y}$. Then the action of the group $T$ on $\tilde{X}$ is without inversion.

The quotient space $X=\tilde{X} / T$ has 3 vertices $p_{0}, p_{1}, p_{2}$, where $p_{0}$ corresponds to the vertices of $\tilde{X}, p_{1}$ to the barycenters of the edges of $\tilde{X}$ and $p_{2}$ to the barycenters of the triangles of $\tilde{X}$. The edges of the quotient space $X$ are $p_{0} p_{1}$, $p_{1} p_{2}$ and $p_{0} p_{1}$. There are 2 triangles $\tau_{1}$ and $\tau_{2}$, and $X$ is topologically a 2 sphere. A fundamental domain for $X$ is drawn in Figure 3.1. 


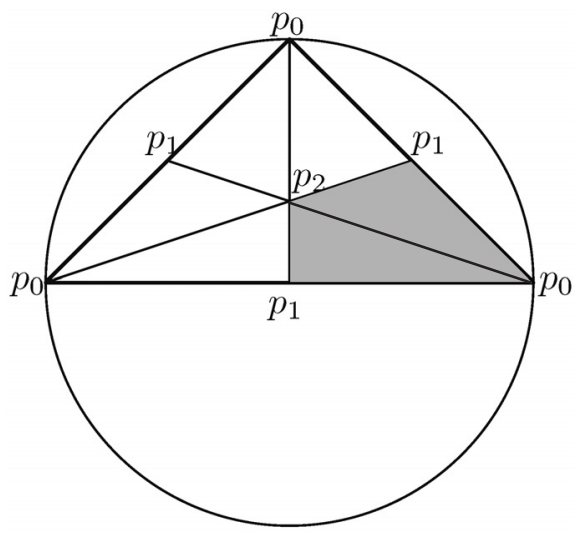

FIGURE 3.1

The isotropy groups of the lifts of a simplex of $X$ are isomorphic. The isomorphism classes of the isotropy groups of the simplices are as follows.

$$
\begin{aligned}
& G_{p_{0}} \cong F, \quad G_{p_{1}} \cong F^{2} \rtimes \mathbf{Z} / 2 \mathbf{Z}, \quad G_{p_{2}} \cong F^{3} \rtimes \mathbf{Z} / 3 \mathbf{Z}, \\
& G_{p_{0} p_{1}} \cong F^{2}, \quad G_{p_{1} p_{2}} \cong F^{3}, \quad G_{p_{0} p_{2}} \cong F^{3}, \\
& G_{\tau_{1}} \cong F^{3}, \quad G_{\tau_{2}} \cong F^{3} .
\end{aligned}
$$

Here the groups $F, F^{2}$ and $F^{3}$ are isomorphic to the subgroups of $T$ fixing 1,2 , and 3 points in $\mathbf{Z}[1 / 2] / \mathbf{Z}$, respectively, and the isotropy groups $G_{p_{1}}$ and $G_{p_{2}}$ permute these points cyclically.

Since the group $F$ is finitely presented (hence so are $F^{2}, F^{3}, F^{2} \rtimes \mathbf{Z} / 2 \mathbf{Z}$, $F^{3} \rtimes \mathbf{Z} / 3 \mathbf{Z}$ ), Finiteness Theorem 3.1 already says that the group $T$ is also finitely presented ([8]).

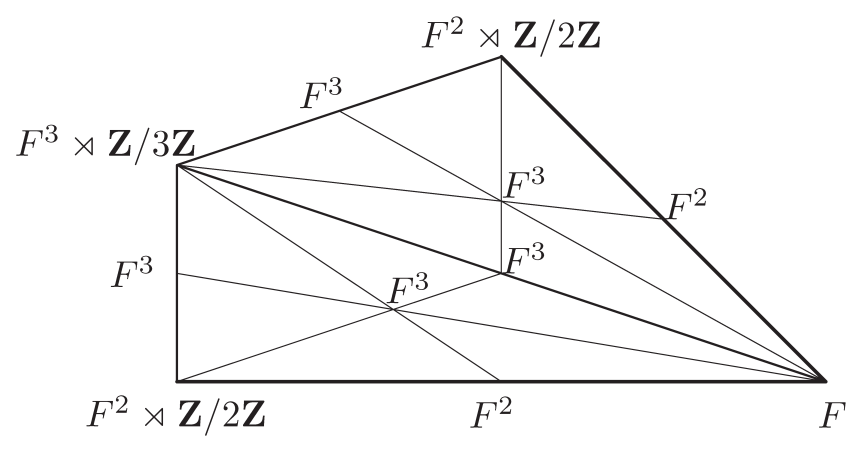

FIGURE 3.2

In order to obtain the presentation of the group $T$, we need the following information on the relationship between the isotropy groups ([8]). 
The group $T$ acts on $\tilde{X}$ without inversion. We consider that the isotropy groups are attached to the vertices of the barycentric subdivision $X^{\prime}$ of $X$. Take a lift of each simplex of $X$ in $\tilde{X}$, or equivalently take a lift $\tilde{p}$ of each vertex $p$ of $X^{\prime}$ in $\tilde{X}^{\prime}$. Then in the group $T$, we have the isotropy subgroups $G_{\tilde{p}}$ of $\tilde{p} \in \tilde{X}^{\prime}$.

Take an edge $p q$ of $X^{\prime}$, where $p$ is a face of $q$ in $X$. Since we lift $q$ to $\tilde{q}, p$ is a face of $q$ in $X$ and the action is without inversion, we have a unique lift $\tilde{p}^{\prime} \tilde{q}$ of the edge $p q$. Then the isotropy subgroup $G_{\tilde{q}}$ is a subgroup of the isotropy subgroup $G_{\tilde{p}^{\prime}}$. The lifted vertex $\tilde{p}^{\prime}$ may be different from $\tilde{p}$, however by taking an element $\eta_{\tilde{p}^{\prime} \tilde{q}}$ of the group $T$ which sends $\tilde{p}^{\prime}$ to $\tilde{p}, \eta_{\tilde{p}^{\prime} \tilde{q}} G_{\tilde{q}} \eta_{\tilde{p}^{\prime} \tilde{q}}^{-1}$ is a subgroup of the isotropy subgroup $G_{\tilde{p}}$.

Let $p q r$ be a triangle of $X^{\prime}$, where $p$ is a face of $q$ in $X$ and $q$ is a face of $r$ in $X$. Since we lift $r$ to $\tilde{r}$ and the action is without inversion, we have a unique lift $\tilde{p}^{\prime \prime} \tilde{q}^{\prime} \tilde{r}$ of the triangle $p q r$, where $\tilde{q}^{\prime} \tilde{r}$ is the previously chosen lift for $q r$ and $\tilde{p}^{\prime \prime} \tilde{r}$ is the previously chosen lift for $p r$. Moreover $\eta_{\tilde{q}^{\prime} \tilde{r}}$ sends $\tilde{p}^{\prime \prime} \tilde{q}^{\prime}$ to $\tilde{p}^{\prime} \tilde{q}$, where $\tilde{p}^{\prime} \tilde{q}$ is the previously chosen lift for $p q$. Now $\eta_{\tilde{p}^{\prime \prime} \tilde{r}} G_{\tilde{r}} \eta_{\tilde{p}^{\prime \prime} \tilde{r}}^{-1}$ and $\eta_{\tilde{p}^{\prime} \tilde{q}} \eta_{\tilde{q}^{\prime} \tilde{r}} G_{\tilde{r}} \eta_{\tilde{q}^{\prime} \tilde{r}^{\prime}}^{-1} \eta_{\tilde{p}^{\prime} \tilde{q}}^{-1}$ are subgroups of the isotropy subgroup $G_{\tilde{p}}$. They are conjugated by $g_{\tilde{p}^{\prime \prime} \tilde{q}^{\prime}, \tilde{q}^{\prime} \tilde{r}=}=$ $\eta_{\tilde{p}^{\prime}} \eta_{\tilde{q}^{\prime} \tilde{r}} \eta_{\tilde{p}^{\prime \prime} \tilde{r}}^{-1} \in T$ which is an element of $G_{\tilde{p}}$.

Now assume that we only know of the information of the isotropy groups and their relationship. That is, assume that we have the presentations of the isotropy groups $G_{p}$ for the vertices $p$ of $X^{\prime}$, the injective homomorphism $\psi_{p q}$ : $G_{q} \rightarrow G_{p}$ for each edge $p q$ of $X^{\prime}$ (which was given by a conjugation by $\eta_{\tilde{p}^{\prime}}$ ), and elements $g_{p q, q r} \in G_{p}$ such that $g_{p q, q r} \psi_{p r} g_{p q, q r}^{-1}=\psi_{p q} \psi_{q r}$ for triangles $p q r$ of $X$.

The complex $X$ with the data $G_{p}, \psi_{p q}, g_{p q, q r}$ is called the complex of groups ([8]). The elements $g_{p q, q r}$ satisfy the cocycle condition ([8]). Then we have the following presentation theorem [8].

Theorem 3.2 (Presentation Theorem [8]). Let $\left(X=\tilde{X} / T, G_{p}, \psi_{p q}, g_{p q, q r}\right)$ be the complex of groups obtained from the action of the group $T$ without inversion on simply connected simplicial complex $\tilde{X}$. Let $\mathbf{T}$ be a maximal tree for the barycentric subdivision $X^{\prime}$. Then a presentation of the group $T$ is given as follows.

Generators of $G_{p}$ for the vertices $p$ of $X^{\prime}$.

Generators $h_{p q}$ corresponding to the oriented edges pq outside of the tree $\mathbf{T}$. Relations of $G_{p}$ for the vertices $p$ of $X^{\prime}$.

Relations coming from the edges; $h_{p q} g h_{p q}^{-1}=\psi_{p q}(g)$ for the generators of $G_{q}$, that is, $h_{p q} g h_{p q}^{-1}$ is written in terms of the generators of $G_{p}$.

Relations coming from the triangles; $g_{p q, q r} h_{p r}=h_{p q} h_{q r}$ for the triangle pqr.

Remark 3.3. If $X$ is not a simplicial complex, then the set of vertices may not determine an edge or a triangle. In practice, however, $X$ would be given as a simplicial complex with identification and we do not meet the ambiguity coming from this fact.

Remark 3.4. We are assuming that $G_{p}$ are presented. For an edge $p q$ of $X^{\prime}$, choosing the lift $\tilde{p}$ to be the lift $\tilde{p}^{\prime}$ which is determined by $\tilde{q}$ is choosing $G_{q}$ to 
be the subgroup of $G_{p}$ and the homomorphism $\psi_{p q}$ to be the inclusion. For the edges $p q$ in the tree $\mathbf{T}$, one can always choose the homomorphism $\psi_{p q}$ to be the inclusion. These simplify the computation.

The complex of groups associated to $X$ usually uses the barycentric subdivision of $X$ as above. In our case, we note that the inclusions $G_{\tau_{1}} \rightarrow G_{p_{0} p_{2}}$ and $G_{\tau_{2}} \rightarrow G_{p_{0} p_{2}}$ can be taken to be the identity. Hence we can use the complex in the following figure (Figure 3.3). The reason will become clear during the actual computation.

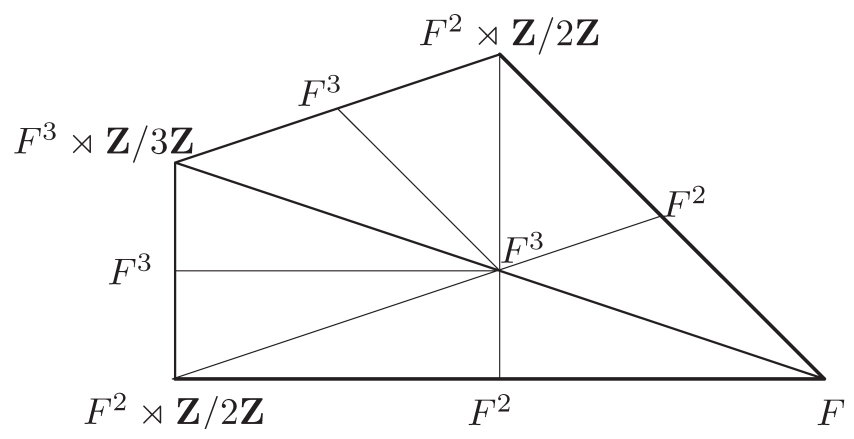

FIGURE 3.3

Now we proceed as follows to give an explicit finite presentation.

We take the triangle $\Delta$ with vertices $0,1 / 4,1 / 2$ in $\tilde{Y}$. See Figure 3.1. We look at the barycentric subdivision of $\Delta$. We take the lift $\tilde{p}_{0}$ of $p_{0}$ to the vertex 0 , then the isotropy group $G_{\tilde{p}_{0}}$ is the isotropy subgroup $T_{0}$ at 0 of the action of the group $T$ and it is identified with the group $F$ generated by $x_{0}$ and $x_{1}$. We lift the 2 triangles $\tau_{1}$ and $\tau_{2}$ to the triangles of the barycentric subdivision of $\Delta$ which has the vertex 0 . Then $p_{0} p_{2}$ is lifted to the common edge $\tilde{p}_{0} \tilde{p}_{2}$. By the choice of the lift $\tilde{p}_{0} \tilde{p}_{2}, G_{\tilde{p}_{0}} \tilde{p}_{2} \cong F^{3}$ is the subgroup $T_{0,1 / 4,1 / 2}$ of the group $T$ which fixes $0,1 / 4$ and $1 / 2$, hence it is generated by $x_{2}, x_{3}, y_{1}, y_{2}, z_{2}, z_{3}$ shown in Figure 3.4.

We choose the lift $\tilde{p}_{0} \tilde{p}_{1}$ of $p_{0} p_{1}$ on the edge joining 0 and $1 / 2$. Then $G_{\tilde{p}_{0} \tilde{p}_{1}} \cong F^{2}$ is the subgroup $T_{0,1 / 2}$ of the group $T$ which fixes 0 and $1 / 2$ and it is generated by $x_{1}, x_{2}, y_{1}, y_{2}$. The group $G_{\tilde{p}_{1} \tilde{p}_{2}} \cong F^{3}$ is also the group $T_{0,1 / 4,1 / 2}$ generated by $x_{2}, x_{3}, y_{1}, y_{2}, z_{2}, z_{3}$.

Let $\rho$ denote the half rotation. Then the isotropy group $G_{\tilde{p}_{1}} \cong F^{2} \rtimes \mathbf{Z} / 2 \mathbf{Z}$ is the subgroup $T_{\{0,1 / 2\}}$ of the group $T$ which fixes the set $\{0,1 / 2\}$ and it is generated by $x_{1}, x_{2}, y_{1}, y_{2}$ and $\rho$.

Let $\omega$ be the element order 3 of the group $T$ defined before. Then $G_{\tilde{p}_{2}} \cong$ $F^{3} \rtimes \mathbf{Z} / 3 \mathbf{Z}$ is the subgroup $T_{\{0,1 / 4,1 / 2\}}$ which fixes the set $\{0,1 / 4,1 / 2\}$ and it is generated by $x_{2}, x_{3}, y_{1}, y_{2}, z_{2}, z_{3}$ and $\omega$.

Let $q_{1}, q_{2}$, and $q_{0}$ denote the barycenters of $\tilde{p}_{0} \tilde{p}_{2}, \tilde{p}_{1} \tilde{p}_{2}$, and $\tilde{p}_{1} \tilde{p}_{2}$, respectively. Put $\tilde{p}_{1}^{\prime}$ to be the other lift of $p_{1}$ on the other triangle, and $q_{0}^{\prime}$ and $q_{2}^{\prime}$ to be the barycenters of $\tilde{p}_{1}^{\prime} \tilde{p}_{2}$ and $\tilde{p}_{0} \tilde{p}_{1}^{\prime}$, respectively. 

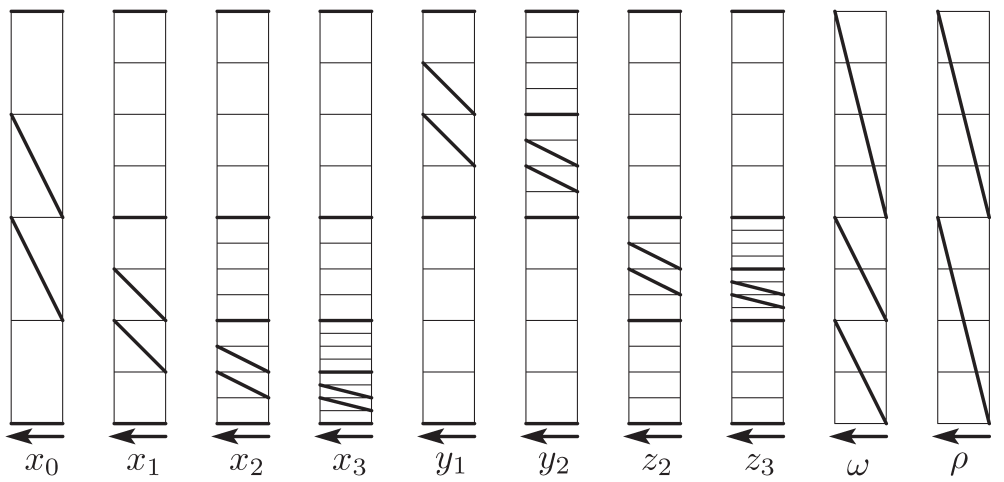

FIGURE 3.4

We take a maximal tree consisting of four edges $\tilde{p}_{2} q_{0}, q_{0} q_{1}, q_{2} q_{1}, \tilde{p}_{0} q_{2}$, $\tilde{p}_{1} q_{2}$. On these edges the corresponding homomorphisms are inclusions.

Note that the edge $\tilde{p}_{2} \tilde{p}_{1}^{\prime}$ is sent to $\tilde{p}_{2} \tilde{p}_{1}$ by $\omega^{-1}$ and the edges $\tilde{p}_{0} \tilde{p}_{1}^{\prime}$ is sent to $\tilde{p}_{0} \tilde{p}_{1}$ by $\rho \omega^{-1}$. Hence $G_{q_{0}^{\prime}}=\omega G_{q_{0}} \omega^{-1}$ and $G_{q_{2}^{\prime}}=\left(\rho \omega^{-1}\right)^{-1} G_{q_{2}} \rho \omega^{-1}$. We use the identification by $\omega^{-1}$ between $G_{\tilde{p}_{1}^{\prime}}$ and $G_{\tilde{p}_{1}} ; G_{\tilde{p}_{1}^{\prime}}=\omega G_{\tilde{p}_{1}} \omega^{-1}$.

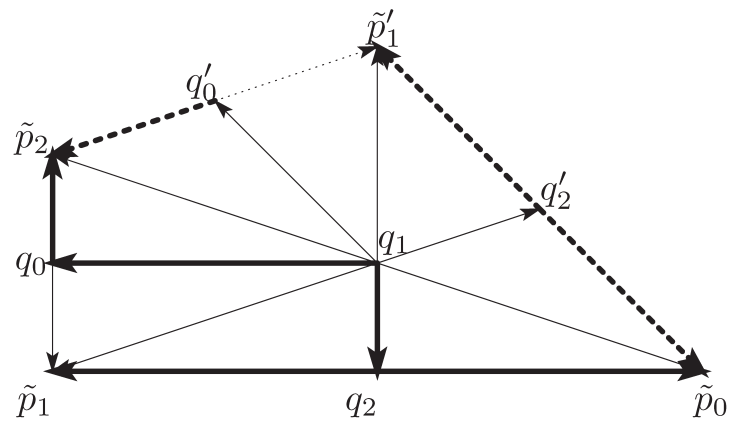

FIGURE 3.5

By Presentation Theorem 3.2, the group $T$ has the following presentation:

Generators of $G_{\tilde{p}_{0}}=T_{0}, G_{\tilde{p}_{1}}=T_{\{0,1 / 2\}}, G_{\tilde{p}_{2}}=T_{\{0,1 / 4,1 / 2\}}$, $G_{q_{0}}=G_{\tilde{p}_{1} \tilde{p}_{2}}=T_{0,1 / 4,1 / 2}, G_{q_{1}}=G_{\tilde{p}_{0} \tilde{p}_{2}}=T_{0,1 / 4,1 / 2}, G_{q_{2}}=G_{\tilde{p}_{0} \tilde{p}_{1}}=T_{0,1 / 2}$.

Generators corresponding to the oriented edges outside of the tree:

$h_{\tilde{p}_{0} q_{1}}, h_{\tilde{p}_{1} q_{1}}, h_{\tilde{p}_{2} q_{1}}, h_{\tilde{p}_{1}^{\prime} q_{1}}, h_{q_{0}^{\prime} q_{1}}, h_{q_{2}^{\prime} q_{1}}, h_{\tilde{p}_{1} q_{0}}$.

Relations for $G_{\tilde{p}_{0}}=T_{0}, G_{\tilde{p}_{1}}=T_{\{0,1 / 2\}}, G_{\tilde{p}_{2}}=T_{\{0,1 / 4,1 / 2\}}$,

$G_{q_{0}}=T_{0,1 / 4,1 / 2}, G_{q_{1}}=T_{0,1 / 4,1 / 2}, G_{q_{2}}=T_{0,1 / 2}$.

Relations coming from the edges:

$g=\psi_{\tilde{p}_{0} q_{2}}(g), g=\psi_{\tilde{p}_{1} q_{2}}(g)$ for the generators $g$ of $G_{q_{2}}$.

$g=\psi_{q_{0} q_{1}}(g), g=\psi_{q_{2} q_{1}}(g)$, 
$h_{\tilde{p}_{0} q_{1}} g h_{\tilde{p}_{0} q_{1}}^{-1}=\psi_{\tilde{p}_{0} q_{1}}(g), h_{\tilde{p}_{1} q_{1}} g h_{\tilde{p}_{1} q_{1}}^{-1}=\psi_{\tilde{p}_{1} q_{1}}(g)$,

$h_{\tilde{p}_{2} q_{1}} g h_{\tilde{p}_{2} q_{1}}^{-1}=\psi_{\tilde{p}_{2} q_{1}}(g), h_{\tilde{p}_{1}^{\prime} q_{1}} g h_{\tilde{p}_{1}^{\prime} q_{1}}^{-1}=\psi_{\tilde{p}_{1}^{\prime} q_{1}}(g)$,

$h_{q_{0}^{\prime} q_{1}} g h_{q_{0}^{\prime} q_{1}}^{-1}=\psi_{q_{0}^{\prime} q_{1}}(g), h_{q_{2}^{\prime} q_{1}} g h_{q_{2}^{\prime} q_{1}}^{-1}=\psi_{q_{2}^{\prime} q_{1}}(g)$ for the generators $g$ of $G_{q_{1}}$.

$g=\psi_{\tilde{p}_{2} q_{0}}(g), h_{\tilde{p}_{1} q_{0}} g h_{\tilde{p}_{1} q_{0}}^{-1}=\psi_{\tilde{p}_{1} q_{0}}(g)$ for the generators $g$ of $G_{q_{0}}$.

Relations coming from the triangles:

$$
\begin{array}{ll}
g_{\tilde{p}_{0} q_{2}, q_{2} q_{1}} h_{\tilde{p}_{0} q_{1}}=1, & g_{\tilde{p}_{1} q_{2}, q_{2} q_{1}} h_{\tilde{p}_{1} q_{1}}=1, \\
g_{\tilde{p}_{1} q_{0}, q_{0} q_{1}} h_{\tilde{p}_{1} q_{1}}=h_{\tilde{p}_{1} q_{0}}, & g_{\tilde{p}_{2} q_{0}, q_{0} q_{1}} h_{\tilde{p}_{2} q_{1}}=1, \\
g_{\tilde{p}_{2} q_{0}^{\prime}, q_{0}^{\prime} q_{1}} h_{\tilde{p}_{2} q_{1}}=h_{q_{0}^{\prime} q_{1}}, & g_{\tilde{p}_{1}^{\prime} q_{0}^{\prime}, q_{0}^{\prime} q_{1}} h_{\tilde{p}_{1}^{\prime} q_{1}}=h_{\tilde{p}_{1} q_{0}} h_{q_{0}^{\prime} q_{1}}, \\
g_{\tilde{p}_{1}^{\prime} q_{2}^{\prime}, q_{2}^{\prime} q_{1}} h_{\tilde{p}_{1}^{\prime} q_{1}}=h_{q_{2}^{\prime} q_{1}}, & g_{\tilde{p}_{0} q_{2}^{\prime}, q_{2}^{\prime} q_{1}} h_{\tilde{p}_{0} q_{1}}=h_{q_{2}^{\prime} q_{1}} .
\end{array}
$$

Here the homomorphisms $\psi_{* *}$ are as follows.

$\psi_{\tilde{p}_{0} q_{2}}$ is the inclusion $T_{0,1 / 2} \subset T_{0}$.

$\psi_{\tilde{p}_{1} q_{2}}$ is the inclusion $T_{0,1 / 2} \subset T_{\{0,1 / 2\}}$.

$\psi_{q_{0} q_{1}}$ is the identity map of $T_{0,1 / 4,1 / 2}$.

$\psi_{q_{2} q_{1}}$ is the inclusion $T_{0,1 / 4,1 / 2} \subset T_{0,1 / 2}$.

$\psi_{\tilde{p}_{0} q_{1}}$ is the inclusion $T_{0,1 / 4,1 / 2} \subset T_{0}$.

$\psi_{\tilde{p}_{1} q_{1}}$ is the inclusion of $T_{0,1 / 4,1 / 2} \subset T_{0,1 / 2} \subset T_{\{0,1 / 2\}}$.

$\psi_{\tilde{p}_{2} q_{1}}$ is the inclusion of $T_{0,1 / 4,1 / 2} \subset T_{\{0,1 / 4,1 / 2\}}$.

$\psi_{\tilde{p}_{1}^{\prime} q_{1}}$ is the composition of $T_{0,1 / 4,1 / 2} \subset T_{\{0,1 / 4\}} \rightarrow T_{\{0,1 / 2\}}$, where the arrow is the conjugation by $\omega^{-1}: \quad g \mapsto \omega^{-1} g \omega$.

$\psi_{q_{0}^{\prime} q_{1}}$ is the outer automorphism of $T_{0,1 / 4,1 / 2}$ given by the conjugation by $\omega^{-1}: \quad g \mapsto \omega^{-1} g \omega$.

$\psi_{q_{2}^{\prime} q_{1}}$ is the composition of $T_{0,1 / 4,1 / 2} \subset T_{0,1 / 4} \rightarrow T_{0,1 / 2}$, where the arrow is the conjugation by $\rho \omega^{-1}: \quad g \mapsto \rho \omega^{-1} g\left(\rho \omega^{-1}\right)^{-1}$.

$\psi_{\tilde{p}_{2} q_{0}}$ is the inclusion $T_{0,1 / 4,1 / 2} \subset T_{\{0,1 / 4,1 / 2\}}$.

$\psi_{\tilde{p}_{1} q_{0}}$ is the inclusion $T_{0,1 / 4,1 / 2} \subset T_{0,1 / 2} \subset T_{\{0,1 / 2\}}$.

The elements $g_{*, *}$ are as follows.

$$
\begin{array}{ll}
g_{\tilde{p}_{0} q_{2}, q_{2} q_{1}}=1 \in T_{0}, & g_{\tilde{p}_{1} q_{2}, q_{2} q_{1}}=1 \in T_{\{0,1 / 2\}}, \\
g_{\tilde{p}_{1} q_{0}, q_{0} q_{1}}=1 \in T_{\{0,1 / 2\}}, & g_{\tilde{p}_{2} q_{0}, q_{0} q_{1}}=1 \in T_{\{0,1 / 4,1 / 2\}}, \\
g_{\tilde{p}_{2} q_{0}^{\prime}, q_{0}^{\prime} q_{1}}=\omega^{-1 \in T_{\{0,1 / 4,1 / 2\}},} & g_{\tilde{p}_{1}^{\prime} q_{0}^{\prime}, q_{0}^{\prime} q_{1}}=1 \in T_{\{0,1 / 2\}}, \\
g_{\tilde{p}_{1}^{\prime} q_{2}^{\prime}, q_{2}^{\prime} q_{1}}=\left(\rho \omega^{-1}\right) \omega=\rho \in T_{\{0,1 / 2\}}, & g_{\tilde{p}_{0} q_{2}^{\prime}, q_{2}^{\prime} q_{1}}=\rho \omega^{-1} \stackrel{=}{=} x_{0} \in T_{0} .
\end{array}
$$

Then by the information on $g_{*, *}$, the elements $h_{*}$ are determined to be 1 except

$$
h_{q_{0}^{\prime} q_{1}}=\omega^{-1}, \quad h_{\tilde{p}_{1}^{\prime} q_{1}}=\omega^{-1}, \quad h_{q_{2}^{\prime} q_{1}}=\rho \omega^{-1}
$$

and we have a relation coming from $g_{*, *}$ :

$$
\rho \omega^{-1}=x_{0} \text {. }
$$

We have the following generators for the group $T$.

$$
x_{0}, x_{1}, x_{2}, x_{3}, y_{1}, y_{2}, z_{2}, z_{3}, \omega, \rho \text {. }
$$

The elements $x_{1}, x_{2}, x_{3}, y_{1}, y_{2}, z_{2}, z_{3}$ belong to different isotropy groups. 
However those inclusions appeared in $\psi_{* *}$ identify the elements $x_{1}, x_{2}, x_{3}, y_{1}, y_{2}$, $z_{2}, z_{3}$ in different isotropy groups.

The relations for $G_{\tilde{p}_{0}}=T_{0}, G_{\tilde{p}_{1}}=T_{\{0,1 / 2\}}, G_{\tilde{p}_{2}}=T_{\{0,1 / 4,1 / 2\}}, G_{q_{0}}=T_{0,1 / 4,1 / 2}$, $G_{q_{1}}=T_{0,1 / 4,1 / 2}, G_{q_{2}}=T_{0,1 / 2}$ are written as follows.

$$
\begin{aligned}
& T_{0}: \quad x_{0}^{-2} x_{1} x_{0}^{2}=x_{1}^{-1} x_{0}^{-1} x_{1} x_{0} x_{1}, \quad x_{0}^{-3} x_{1} x_{0}^{3}=x_{1}^{-1} x_{0}^{-2} x_{1} x_{0}^{2} x_{1} . \\
& T_{0,1 / 2}: \quad x_{1}^{-2} x_{2} x_{1}^{2}=x_{2}^{-1} x_{1}^{-1} x_{2} x_{1} x_{2}, \quad x_{1}^{-3} x_{2} x_{1}^{3}=x_{2}^{-1} x_{1}^{-2} x_{2} x_{1}^{2} x_{2}, \\
& y_{1}^{-2} y_{2} y_{1}^{2}=y_{2}^{-1} y_{1}^{-1} y_{2} y_{1} y_{2}, \quad y_{1}^{-3} y_{2} y_{1}^{3}=y_{2}^{-1} y_{1}^{-2} y_{2} y_{1}^{2} y_{2}, \\
& x_{1} y_{1}=y_{1} x_{1}, \quad x_{1} y_{2}=y_{2} x_{1}, \quad x_{2} y_{1}=y_{1} x_{2}, \quad x_{2} y_{2}=y_{2} x_{2} \text {. } \\
& T_{0,1 / 4,1 / 2}: \quad x_{2}^{-2} x_{3} x_{2}^{2}=x_{3}^{-1} x_{2}^{-1} x_{3} x_{2} x_{3}, \quad x_{2}^{-3} x_{3} x_{2}^{3}=x_{3}^{-1} x_{2}^{-2} x_{3} x_{2}^{2} x_{3} \text {, } \\
& y_{1}^{-2} y_{2} y_{1}^{2}=y_{2}^{-1} y_{1}^{-1} y_{2} y_{1} y_{2}, \quad y_{1}^{-3} y_{2} y_{1}^{3}=y_{2}^{-1} y_{1}^{-2} y_{2} y_{1}^{2} y_{2} \text {. } \\
& z_{2}^{-2} z_{3} z_{2}^{2}=z_{3}^{-1} z_{2}^{-1} z_{3} z_{2} z_{3}, \quad z_{2}^{-3} z_{3} z_{2}^{3}=z_{3}^{-1} z_{2}^{-2} z_{3} z_{2}^{2} z_{3}, \\
& x_{1} y_{1}=y_{1} x_{1}, \quad x_{1} y_{2}=y_{2} x_{1}, \quad x_{2} y_{1}=y_{1} x_{2}, \quad x_{2} y_{2}=y_{2} x_{2}, \\
& y_{1} z_{1}=z_{1} y_{1}, \quad y_{1} z_{2}=z_{2} y_{1}, \quad y_{2} z_{1}=z_{1} y_{2}, \quad y_{2} z_{2}=z_{2} y_{2}, \\
& x_{1} z_{1}=z_{1} x_{1}, \quad x_{1} z_{2}=z_{2} x_{1}, \quad x_{2} z_{1}=z_{1} x_{2}, \quad x_{2} z_{2}=z_{2} x_{2} . \\
& T_{\{0,1 / 2\}}: \quad x_{1}^{-2} x_{2} x_{1}^{2}=x_{2}^{-1} x_{1}^{-1} x_{2} x_{1} x_{2}, \quad x_{1}^{-3} x_{2} x_{1}^{3}=x_{2}^{-1} x_{1}^{-2} x_{2} x_{1}^{2} x_{2} \text {, } \\
& \rho^{2}=1, \quad \rho x_{1} \rho=y_{1}, \quad \rho x_{2} \rho=y_{2}, \\
& x_{1} y_{1}=y_{1} x_{1}, \quad x_{1} y_{2}=y_{2} x_{1}, \quad x_{2} y_{1}=y_{1} x_{2}, \quad x_{2} y_{2}=y_{2} x_{2} \text {. } \\
& T_{\{0,1 / 4,1 / 2\}}: \quad x_{2}^{-2} x_{3} x_{2}^{2}=x_{3}^{-1} x_{2}^{-1} x_{3} x_{2} x_{3}, \quad x_{2}^{-3} x_{3} x_{2}^{3}=x_{3}^{-1} x_{2}^{-2} x_{3} x_{2}^{2} x_{3} \text {, } \\
& \omega^{3}=1, \omega^{-1} x_{2} \omega=y_{1}, \omega^{-1} x_{3} \omega=y_{2}, \omega x_{2} \omega^{-1}=z_{2}, \omega x_{3} \omega^{-1}=z_{3}, \\
& x_{1} y_{1}=y_{1} x_{1}, \quad x_{1} y_{2}=y_{2} x_{1}, \quad x_{2} y_{1}=y_{1} x_{2}, \quad x_{2} y_{2}=y_{2} x_{2}, \\
& y_{1} z_{1}=z_{1} y_{1}, \quad y_{1} z_{2}=z_{2} y_{1}, \quad y_{2} z_{1}=z_{1} y_{2}, \quad y_{2} z_{2}=z_{2} y_{2}, \\
& x_{1} z_{1}=z_{1} x_{1}, \quad x_{1} z_{2}=z_{2} x_{1}, \quad x_{2} z_{1}=z_{1} x_{2}, \quad x_{2} z_{2}=z_{2} x_{2} .
\end{aligned}
$$

Strictly speaking, we should use different letters for different groups. Using the same letters is justified by the inclusions in the following relations coming from the edges. In the following, the letters in the left-hand-sides and the letters in the right-hand-sides are in the different groups. However the following relations justify that they are identified.

$$
\begin{aligned}
& \psi_{\tilde{p}_{0} q_{2}}: x_{1}=x_{1}, \quad x_{2}=x_{0}^{-1} x_{1} x_{0}, \\
& y_{1}=x_{0}^{2} x_{1}^{-1} x_{0}^{-1}, \quad y_{2}=x_{0} x_{1}^{2} x_{0}^{-1} x_{1}^{-1} x_{0} x_{1}^{-1} x_{0}^{-1} . \\
& \psi_{\tilde{p}_{1} q_{2}}: \quad x_{1}=x_{1}, \quad x_{2}=x_{2}, \quad y_{1}=y_{1}, \quad y_{2}=y_{2}, \\
& \psi_{q_{0} q_{1}}: \quad x_{2}=x_{2}, \quad x_{3}=x_{3}, \quad y_{1}=y_{1}, \quad y_{2}=y_{2}, \quad z_{2}=z_{2}, \quad z_{3}=z_{3} .
\end{aligned}
$$




$$
\begin{aligned}
& \psi_{q_{2} q_{1}}: \quad x_{2}=x_{2}, \quad x_{3}=x_{1}^{-1} x_{2} x_{1}, \quad y_{1}=y_{1}, \quad y_{2}=y_{2}, \\
& z_{2}=x_{1}^{2} x_{2}^{-1} x_{1}^{-1}, \quad z_{3}=x_{1} x_{2}^{2} x_{1}^{-1} x_{2}^{-1} x_{1} x_{2}^{-1} x_{1}^{-1} . \\
& \psi_{\tilde{p}_{0} q_{1}}: \quad x_{2}=x_{0}^{-1} x_{1} x_{0}, \quad x_{3}=x_{0}^{-2} x_{1} x_{0}^{2} \text {, } \\
& y_{1}=x_{0}^{2} x_{1}^{-1} x_{0}^{-1}, \quad y_{2}=x_{0} x_{1}^{2} x_{0}^{-1} x_{1}^{-1} x_{0} x_{1}^{-1} x_{0}^{-1}, \\
& z_{2}=x_{1}^{2} x_{0}^{-1} x_{1}^{-1} x_{0} x_{1}^{-1}, \quad z_{3}=x_{1} x_{0}^{-1} x_{1}^{2} x_{0}^{-1} x_{1}^{-1} x_{0} x_{1}^{-1} x_{0} x_{1}^{-1} \text {. } \\
& \psi_{\tilde{p}_{1} q_{1}}: \quad x_{2}=x_{2}, \quad x_{3}=x_{1}^{-1} x_{2} x_{1}, \quad y_{1}=y_{1}, \quad y_{2}=y_{2} \text {, } \\
& z_{2}=x_{1}^{2} x_{2}^{-1} x_{1}^{-1}, \quad z_{3}=x_{1} x_{2}^{2} x_{1}^{-1} x_{2}^{-1} x_{1} x_{2}^{-1} x_{1}^{-1} \text {. } \\
& \psi_{\tilde{p}_{2} q_{1}}: \quad x_{2}=x_{2}, \quad x_{3}=x_{3}, \quad y_{1}=y_{1}, \quad y_{2}=y_{2}, \quad z_{2}=z_{2}, \quad z_{3}=z_{3} . \\
& \psi_{\tilde{p}_{1}^{\prime} q_{1}}: \quad \omega^{-1} x_{2} \omega=y_{1}, \quad \omega^{-1} x_{3} \omega=y_{2}, \quad \omega^{-1} y_{1} \omega=z_{2}, \quad \omega^{-1} y_{2} \omega=z_{3} \text {, } \\
& \omega^{-1} z_{2} \omega=x_{2}, \quad \omega^{-1} z_{3} \omega=x_{3} . \\
& \psi_{q_{0}^{\prime} q_{1}}: \quad \omega^{-1} x_{2} \omega=y_{1}, \quad \omega^{-1} x_{3} \omega=y_{2}, \quad \omega^{-1} y_{1} \omega=z_{2}, \quad \omega^{-1} y_{2} \omega=z_{3} \text {, } \\
& \omega^{-1} z_{2} \omega=x_{2}, \quad \omega^{-1} z_{3} \omega=x_{3} . \\
& \psi_{q_{2}^{\prime} q_{1}}: \quad \rho \omega^{-1} x_{2}\left(\rho \omega^{-1}\right)^{-1}=x_{1}, \quad \rho \omega^{-1} x_{3}\left(\rho \omega^{-1}\right)^{-1}=x_{2}, \\
& \rho \omega^{-1} y_{1}\left(\rho \omega^{-1}\right)^{-1}=y_{1}^{2} y_{2}^{-1} y_{1}^{-1}, \\
& \rho \omega^{-1} y_{2}\left(\rho \omega^{-1}\right)^{-1}=y_{1} y_{2}^{2} y_{1}^{-1} y_{2}^{-1} y_{1} y_{2}^{-1} y_{1}^{-1} \text {, } \\
& \rho \omega^{-1} z_{2}\left(\rho \omega^{-1}\right)^{-1}=y_{2}, \quad \rho \omega^{-1} z_{3}\left(\rho \omega^{-1}\right)^{-1}=y_{1}^{-1} y_{2} y_{1} . \\
& \psi_{\tilde{p}_{2} q_{0}}: \quad x_{2}=x_{2}, \quad x_{3}=x_{3}, \quad y_{1}=y_{1}, \quad y_{2}=y_{2}, \quad z_{2}=z_{2}, \quad z_{3}=z_{3} . \\
& \psi_{\tilde{p}_{1} q_{0}}: \quad x_{2}=x_{2}, \quad x_{3}=x_{1}^{-1} x_{2} x_{1}, \quad y_{1}=y_{1}, \quad y_{2}=y_{2}, \\
& z_{2}=x_{1}^{2} x_{2}^{-1} x_{1}^{-1}, \quad z_{3}=x_{1} x_{2}^{2} x_{1}^{-1} x_{2}^{-1} x_{1} x_{2}^{-1} x_{1}^{-1} .
\end{aligned}
$$

Here the way of writing $y_{2}, z_{2}$ and $z_{3}$ by the generators of different groups are drawn in Figures 3.6, 3.7 and 3.8.

In the presentation of the group $T$, we have the presentation of the group $F=T_{0}$. We know that the group $F$ is isomorphic to the dyadic piecewise linear homeomorphisms of the interval $[0,1]$. By defining $x_{2}, x_{3}, y_{1}, y_{2}, z_{2}, z_{3}$ in term of $x_{0}$ and $x_{1}$, we know that the relations in $T_{0,1 / 2}$ or $T_{0,1 / 4,1 / 2}$ are derived from the two relations of the group $F$. Hence those relations in terms of $x_{0}, x_{1}, x_{2}$, $x_{3}, y_{1}, y_{2}, z_{2}, z_{3}$ follow from those of the group $F$. By using $\rho \omega^{-1}=x_{0}$, the relations in terms of $x_{2}, x_{3}, y_{1}, y_{2}, z_{2}, z_{3}$ and $\rho \omega^{-1}$ are also derived from the relations of the group $F$. Thus we have the following list of possibly nontrivial relations.

$$
\begin{aligned}
& x_{2}=x_{0}^{-1} x_{1} x_{0}, x_{3}=x_{1}^{-1} x_{2} x_{1}, x_{0}^{-1} x_{2} x_{0}=x_{3}, x_{0}^{-1} x_{3} x_{0}=x_{1}^{-1} x_{3} x_{1}, \\
& y_{1}=x_{0}^{2} x_{1}^{-1} x_{0}^{-1}, y_{2}=x_{0} x_{1}^{2} x_{0}^{-1} x_{1}^{-1} x_{0} x_{1}^{-1} x_{0}^{-1},
\end{aligned}
$$


GROUP GENERATED BY HALF TRANSVECTIONS

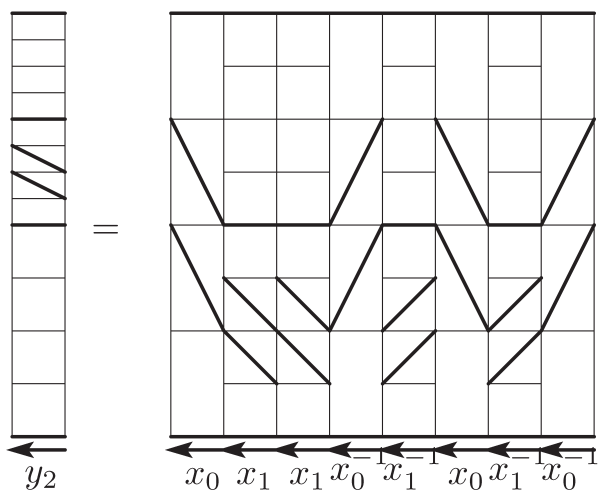

FIGURE 3.6
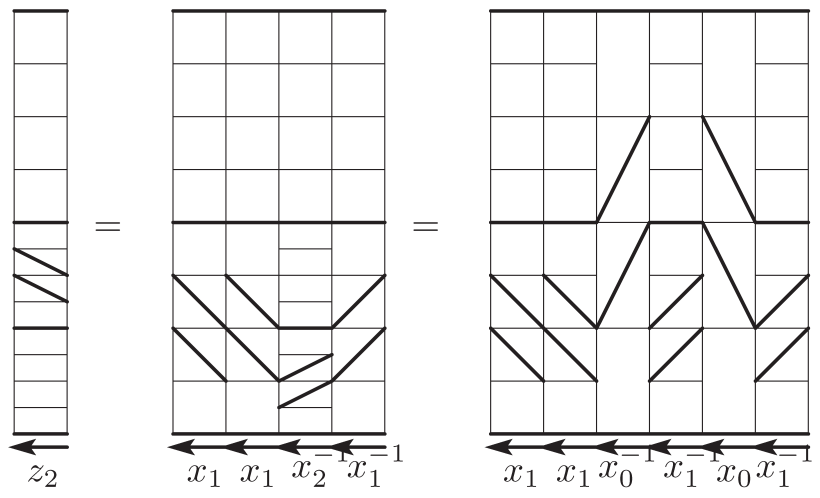

FIGURE 3.7
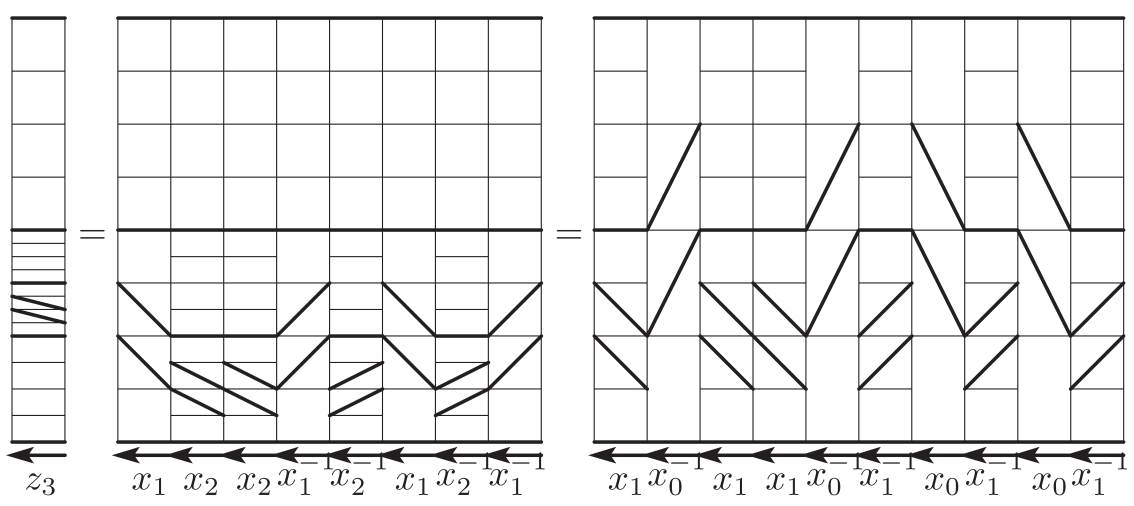

FIGURE 3.8 


$$
\begin{aligned}
& z_{2}=x_{1}^{2} x_{2}^{-1} x_{1}^{-1}, z_{3}=x_{1} x_{2}^{2} x_{1}^{-1} x_{2}^{-1} x_{1} x_{2}^{-1} x_{1}^{-1} \\
& \rho^{2}=1, \omega^{3}=1, \rho \omega^{-1}=x_{0}, \rho x_{1} \rho=y_{1}, \rho x_{2} \rho=y_{2}, \\
& y_{1}=\omega^{-1} x_{2} \omega, y_{2}=\omega^{-1} x_{3} \omega, z_{2}=\omega^{-1} y_{1} \omega, z_{3}=\omega^{-1} y_{2} \omega .
\end{aligned}
$$

Now we show that the first 3 lines of the above relations together with $\rho^{2}=1, \omega^{3}=1, \rho \omega^{-1}=x_{0}, \rho x_{1} \rho=y_{1}$ and $z_{2}=\omega^{-1} y_{1} \omega$ imply $y_{1}=\omega^{-1} x_{2} \omega$, $\rho x_{2} \rho=y_{2}, y_{2}=\omega^{-1} x_{3} \omega$ and $z_{3}=\omega^{-1} y_{2} \omega$.

In fact, noticing $\rho^{2}=x_{0} \omega x_{0} \omega=1$,

$$
\omega y_{1}=\omega\left(x_{0} \omega\right) x_{1}\left(x_{0} \omega\right)=x_{0}^{-1} x_{1}\left(x_{0} \omega\right)=x_{2} \omega .
$$

Using this,

$$
\begin{aligned}
\rho x_{2} \rho & =x_{0} \omega x_{2} x_{0} \omega=x_{0} \omega x_{2} \omega^{-1} \omega x_{0} \omega \\
& =x_{0} \omega^{2} y_{1} \omega^{-2} x_{0}^{-1}=x_{0} \omega^{-1} y_{1} \omega x_{0}^{-1} \\
& =x_{0} z_{2} x_{0}^{-1}=y_{2} .
\end{aligned}
$$

Then,

$$
\omega y_{2}=\omega\left(x_{0} \omega\right) x_{2}\left(x_{0} \omega\right)=x_{0}^{-1} x_{2}\left(x_{0} \omega\right)=x_{3} \omega
$$

Since

$$
\omega x_{1} \omega^{-1}=x_{0}^{-1} \rho x_{1} \rho x_{0}=x_{0}^{-1} y_{1} x_{0}=x_{0}^{-1} x_{0}^{2} x_{1}^{-1} x_{0}^{-1} x_{0}=x_{0} x_{1}^{-1},
$$

we have

$$
\begin{aligned}
\omega^{-1} y_{2} \omega & =\omega^{-1} x_{0} z_{2} x_{0}^{-1} \omega=\omega^{-1} x_{0} \omega \omega^{-1} z_{2} \omega \omega^{-1} x_{0}^{-1} \omega \\
& =\omega x_{0}^{-1} x_{2} x_{0} \omega^{-1}=\omega x_{1}^{-1} x_{2} x_{1} \omega^{-1} \\
& =\omega x_{1}^{-1} \omega^{-1} \omega x_{2} \omega^{-1} \omega x_{1} \omega^{-1} \\
& =x_{1} x_{0}^{-1} z_{2} x_{0} x_{1}^{-1}=z_{3} .
\end{aligned}
$$

Thus we showed the following theorem.

THEOREM 3.5 [4]. The Higman-Thompson group $T$ is presented as follows.

Generators:

$x_{0}, x_{1}, x_{2}, x_{3}, y_{1}, z_{2}, \omega, \rho$.

Relations:

$x_{2}=x_{0}^{-1} x_{1} x_{0}, x_{3}=x_{1}^{-1} x_{2} x_{1}, x_{0}^{-1} x_{2} x_{0}=x_{3}, x_{0}^{-1} x_{3} x_{0}=x_{1}^{-1} x_{3} x_{1}$,

$y_{1}=x_{0}^{2} x_{1}^{-1} x_{0}^{-1}, z_{2}=x_{1}^{2} x_{2}^{-1} x_{1}^{-1}$,

$\rho^{2}=1, \omega^{3}=1, \rho \omega^{-1}=x_{0}, \rho x_{1} \rho=y_{1}, z_{2}=\omega^{-1} y_{1} \omega$.

Remark 3.6. A finite presentation of the group $T$ is given in [4], where it is obtained by solving a word problem. Our presentation is equivalent to that in [4]. Fortunately, the generators $A, B$ and $C$ of [4] are $\imath x_{0} l, \imath x_{1} l$ and $\imath \omega \iota$, respectively, where $l:[0,1] /\{0,1\} \rightarrow[0,1] /\{0,1\}$ is the orientation reversing 
homeomorphism $l(x)=1-x$. Hence the relations in [4] is translated to the relations in $x_{0}, x_{1}, \omega$ by just substituting $A=x_{0}, B=x_{1}, C=\omega$. Then their relations 1) and 2) are, as the relation of $F$, equivalent to $x_{2}=x_{0}^{-1} x_{1} x_{0}, x_{3}=$ $x_{1}^{-1} x_{2} x_{1}, x_{0}^{-1} x_{2} x_{0}=x_{3}, x_{0}^{-1} x_{3} x_{0}=x_{1}^{-1} x_{3} x_{1}$, which is also noted in [4]. Their relation 3) is $\omega x_{1} \omega^{-1}=x_{0} x_{1}^{-1}$, and it is derived from $\rho=x_{0} \omega, \rho x_{1} \rho=y_{1}$ and $y_{1}=x_{0}^{2} x_{1}^{-1} x_{0}^{-1}$ as we showed. Their relation 4) is $x_{0}^{-1} \omega x_{1} x_{0}^{-1} x_{1} x_{0}=x_{1} x_{0}^{-2} \omega x_{1}^{2}$, and this is exactly the same formula obtained from $z_{2}=\omega^{-1} y_{1} \omega$ by substituting $y_{1}=x_{0}^{2} x_{1}^{-1} x_{0}^{-1}, z_{2}=x_{1}^{2} x_{0}^{-1} x_{1}^{-1} x_{0} x_{1}^{-1}$, i.e., $x_{1}^{2} x_{0}^{-1} x_{1}^{-1} x_{0} x_{1}^{-1}=\omega^{-1} x_{0}^{2} x_{1}^{-1} x_{0}^{-1} \omega$. Their relation 5) is $\omega x_{0}=x_{0}^{-1} \omega x_{1} x_{0}^{-1} \omega x_{1}$, and this is obtained by $1=\rho^{2}=$ $x_{0} \omega x_{0} \omega$ and relation 3). Their relation 6) is just $\omega^{3}=1$. Conversely, their relations imply our relations. In fact, 1) and 2) are equivalent to our first line. Their 3), 5) and 6) imply $\omega x_{0}=x_{0}^{-1} \omega x_{1} x_{0}^{-1} \omega x_{1}=x_{0}^{-1} \omega x_{1} x_{0}^{-1} x_{0} x_{1}^{-1} \omega=x_{0}^{-1} \omega \omega=$ $x_{0}^{-1} \omega^{-1}$. Hence by defining $\rho=x_{0} \omega$ we have $\rho \rho=1$. Then by defining $y_{1}=\rho x_{1} \rho$, we obtain $y_{1}=x_{0} \omega x_{1} x_{0} \omega=x_{0} x_{0} x_{1}^{-1} \omega x_{0} \omega x_{0} x_{0} x_{1}^{-1} x_{0}^{-1} \rho \rho=x_{0} x_{0} x_{1}^{-1} x_{0}^{-1}$. Now their 4) is $x_{0}^{-1} \omega x_{1} x_{0}^{-1} x_{1} x_{0}=x_{1} x_{0}^{-2} \omega x_{1}^{2}$ or $\omega^{-1} x_{0} x_{0} x_{1}^{-1} x_{0}^{-1} \omega=x_{1}^{2} x_{0}^{-1} x_{1}^{-1} x_{0} x_{1}^{-1}$, and we obtain $z_{2}=\omega^{-1} y_{1} \omega=\omega^{-1} x_{0} x_{0} x_{1}^{-1} x_{0}^{-1} \omega=x_{1}^{2} x_{0}^{-1} x_{1}^{-1} x_{0} x_{1}^{-1}=x_{1}^{2} x_{2}^{-1} x_{1}^{-1}$.

Remark 3.7. A finite presentation of the group $T$ with respect to $\omega$ and $\eta$ is written down in [10] which is derived from the presentation given in [4]. See also $\$ 4$.

\section{A finite presentation of the group generated by half transvections}

It may be interesting to write down the presentation of the group $G$ generated by half transvections for the generators $x_{1}, y_{1}, u_{1}, v_{1}$. The presentation is obtained from Theorem 3.5 by defining $u_{1}$ and $v_{1}$ in terms of the generators of the group $T$. We define $\eta=\omega x_{1} x_{0}^{-1}$ which is the quarter rotation to do this. Then we have

\section{Generators:}

$x_{0}, x_{1}, x_{2}, x_{3}, y_{1}, z_{2}, \omega, \rho, \eta, u_{1}, v_{1}$.

Relations:

$x_{2}=x_{0}^{-1} x_{1} x_{0}, x_{3}=x_{1}^{-1} x_{2} x_{1}, x_{0}^{-1} x_{2} x_{0}=x_{3}, x_{0}^{-1} x_{3} x_{0}=x_{1}^{-1} x_{3} x_{1}$,

$y_{1}=x_{0}^{2} x_{1}^{-1} x_{0}^{-1}, z_{2}=x_{1}^{2} x_{2}^{-1} x_{1}^{-1}$,

$\rho^{2}=1, \omega^{3}=1, \rho \omega^{-1}=x_{0}, \rho x_{1} \rho=y_{1}, z_{2}=\omega^{-1} y_{1} \omega$,

$\eta=\omega x_{1} x_{0}^{-1}, u_{1}=\eta x_{1} \eta^{-1}, v_{1}=\eta y_{1} \eta^{-1}$. we have

Using $\eta=\omega x_{1} x_{0}^{-1}, \rho \omega^{-1}=x_{0}, \rho x_{1} \rho=y_{1}, \rho^{2}=1, y_{1}=x_{0}^{2} x_{1}^{-1} x_{0}^{-1}$ and $\omega^{3}=1$,

$$
\begin{aligned}
\eta^{2} & =\omega x_{1} x_{0}^{-1} \omega x_{1} x_{0}^{-1}=\omega x_{1} x_{0}^{-1} x_{0}^{-1} \rho x_{1} x_{0}^{-1} \\
& =\omega x_{1} x_{0}^{-1} x_{0}^{-1} y_{1} \rho x_{0}^{-1}=\omega x_{0}^{-1} \rho x_{0}^{-1}=\omega \omega x_{0}^{-1} \\
& =\omega^{-1} x_{0}^{-1}=\rho^{-1}=\rho .
\end{aligned}
$$

Then we can replace the relation $\omega^{3}=1$ which is used only once in the 
computation by $\eta^{2}=\rho$. We substitute $x_{2}=x_{0}^{-1} x_{1} x_{0}$ and $x_{3}=x_{1}^{-1} x_{2} x_{1}$ to $x_{0}^{-1} x_{2} x_{0}=x_{3}$ and obtain

$$
x_{0}^{-1} x_{0}^{-1} x_{1} x_{0} x_{0}=x_{1}^{-1} x_{0}^{-1} x_{1} x_{0} x_{1}
$$

which reads

$$
x_{1} y_{1}=y_{1} x_{1}
$$

by using $y_{1}=x_{0}^{2} x_{1}^{-1} x_{0}^{-1}$. We substitute $x_{3}=x_{1}^{-1} x_{2} x_{1}$ to $x_{0}^{-1} x_{3} x_{0}=x_{1}^{-1} x_{3} x_{1}$ and obtain

$$
x_{0}^{-1} x_{0}^{-1} x_{2} x_{0} x_{0}=x_{1}^{-1} x_{0}^{-1} x_{2} x_{0} x_{1}
$$

which reads

$$
x_{2} y_{1}=y_{1} x_{2} \text {. }
$$

By substituting $x_{0}=\eta^{-1} \omega x_{1}$ to $y_{1}=x_{0}^{2} x_{1}^{-1} x_{0}^{-1}$, we have

$$
y_{1}=\eta^{-1} \omega x_{1} \eta^{-1} \omega x_{1} x_{1}^{-1} x_{1}^{-1} \omega^{-1} \eta=\eta^{-1} \omega x_{1} \eta^{-1} \omega x_{1}^{-1} \omega^{-1} \eta,
$$

that is,

$$
v_{1}=\eta y_{1} \eta^{-1}=\omega x_{1} \eta^{-1} \omega x_{1}^{-1} \omega^{-1}
$$

$x_{2}$ is written as

$$
x_{2}=x_{0}^{-1} x_{1} x_{0}=x_{1}^{-1} \omega^{-1} \eta x_{1} \eta^{-1} \omega x_{1} .
$$

Then $y_{1} x_{2}=x_{2} y_{1}$ is written as

$$
x_{1}^{-1} \omega^{-1} \eta x_{1} \eta^{-1} \omega x_{1} y_{1}=y_{1} x_{1}^{-1} \omega^{-1} \eta x_{1} \eta^{-1} \omega x_{1},
$$

which is

$$
\omega^{-1} u_{1} \omega y_{1}=y_{1} \omega^{-1} u_{1} \omega
$$

Then

that is,

$$
\begin{aligned}
\omega^{-1} y_{1} \omega & =z_{2}=x_{1}^{2} x_{2}^{-1} x_{1}^{-1} \\
& =x_{1}^{2} x_{1}^{-1} \omega^{-1} \eta x_{1}^{-1} \eta^{-1} \omega x_{1} x_{1}^{-1} \\
& =x_{1} \omega^{-1} u_{1}^{-1} \omega
\end{aligned}
$$

$$
y_{1}=\omega x_{1} \omega^{-1} u_{1}^{-1} .
$$

Thus we obtain the following presentation of the group $T$.

\section{Generators:}

$x_{1}, y_{1}, \omega, \rho, \eta, u_{1}, v_{1}$.

Relations:

$$
\begin{aligned}
& x_{1} y_{1}=y_{1} x_{1}, \omega^{-1} u_{1} \omega y_{1}=y_{1} \omega^{-1} u_{1} \omega, \\
& v_{1}=\omega x_{1} \eta^{-1} \omega x_{1}^{-1} \omega^{-1}, \\
& \rho^{2}=1, \rho=\eta^{2}, \omega^{-1}=\eta \omega x_{1}, \rho x_{1} \rho=y_{1},
\end{aligned}
$$


$y_{1}=\omega x_{1} \omega^{-1} u_{1}^{-1}$

$u_{1}=\eta x_{1} \eta^{-1}, v_{1}=\eta y_{1} \eta^{-1}$.

Since $\eta^{-1} \omega^{-1}=\omega x_{1}=y_{1} u_{1} \omega$ and $\omega^{3}=1$ is shown by the above relations,

$$
\omega=\omega^{-2}=\eta y_{1} u_{1} \text {. }
$$

This replaces $y_{1}=\omega x_{1} \omega^{-1} u_{1}^{-1}$. By substituting $\omega=\eta y_{1} u_{1}$ and $\rho=\eta^{2}$, we obtain the following presentation.

\section{Generators:}

$x_{1}, y_{1}, \eta, u_{1}, v_{1}$.

Relations:

$x_{1} y_{1}=y_{1} x_{1}, u_{1}^{-1} y_{1}^{-1} \eta^{-1} u_{1} \eta y_{1} u_{1} y_{1}=y_{1} u_{1}^{-1} y_{1}^{-1} \eta^{-1} u_{1} \eta y_{1} u_{1}$,

$v_{1}=\eta y_{1} u_{1} x_{1} y_{1} u_{1} x_{1}^{-1} u_{1}^{-1} y_{1}^{-1} \eta^{-1}$

$\eta^{4}=1, \rho u_{1}^{-1} y_{1}^{-1} \eta^{-1}=y_{1} u_{1} x_{1}, \eta^{2} x_{1} \eta^{-2}=y_{1}$,

$u_{1}=\eta x_{1} \eta^{-1}, v_{1}=\eta y_{1} \eta^{-1}$.

Now using $u_{1}=\eta x_{1} \eta^{-1}, y_{1}=\eta u_{1} \eta^{-1}, v_{1}=\eta y_{1} \eta^{-1}$, we move $\eta$ or $\eta^{-1}$ to the end of the words, and we obtain the representation in the following theorem.

THEOREM 4.1. The Higman-Thompson group $T$ is presented as follows.

\section{Generators:}

$x_{1}, y_{1}, u_{1}, v_{1}, \eta$.

Relations:

$x_{1} y_{1}=y_{1} x_{1}, u_{1}^{-1} x_{1} u_{1} y_{1}=y_{1} u_{1}^{-1} x_{1} u_{1}, \quad y_{1} u_{1} x_{1}=u_{1} x_{1} y_{1} u_{1}$

$\eta=x_{1} v_{1} y_{1} u_{1} x_{1}, \eta^{4}=1, u_{1}=\eta x_{1} \eta^{-1}, y_{1}=\eta u_{1} \eta^{-1}, v_{1}=\eta y_{1} \eta^{-1}$.

Here $x_{1}, y_{1}, u_{1}, v_{1}$ are the half transvections such that $x_{1} y_{1}$ and $u_{1} v_{1}$ corresponds to the actions of $\left(\begin{array}{ll}1 & 1 \\ 0 & 1\end{array}\right)$ and $\left(\begin{array}{cc}1 & 0 \\ -1 & 1\end{array}\right)$ of $S L(2 ; \mathbf{R})$ on the set of rays of $\mathbf{R}^{2}$, and $\eta$ corresponds to the quarter rotation.

\section{REFERENCES}

[1] A. Banyaga, The structure of classical diffeomorphism groups, Mathematics and its Applications 400, Kluwer Academic Publishers Group, Dordrecht, 1997.

[2] K. S. Brown, Finiteness properties of groups, Proceedings of the Northwestern conference on cohomology of groups (Evanston, Ill., 1985), J. Pure Appl. Algebra 44 (1987), 4575.

[3] K. S. Brown, The geometry of finitely presented infinite simple groups, Algorithms and classification in combinatorial group theory (Berkeley, CA, 1989), Math. Sci. Res. Inst. Publ. 23, Springer, New York, 1992, 121-136.

[4] J. W. Cannon, W. J. Floyd and W. R. Parry, Introductory notes on Richard Thompson's groups, L'Enseignement Mathématique 42 (1996), 215-256.

[5] D. B. A. Epstein, The simplicity of certain groups of homeomorphisms, Compositio Math. 22 (1970), 165-173. 
[6] E. Ghys et V. Sergiescu, Sur un groupe remarquable de difféomorphismes du cercle, Comm. Math. Helv. 62 (1987), 185-239.

[ 7 ] P. GreEnBerg, Projective aspects of the Higman-Thompson group, Group theory from a geometrical view point, Trieste, 1990, World Scientific, 1991, 633-644.

[8] A. Haefliger, Complexes of groups and orbihedra, Group theory from a geometrical viewpoint, Trieste, 1990, World Scientific, 1991, 504-540.

[9] M. Imbert, Sur l'isomorphisme du groupe de Richard Thompson avec le groupe de Ptolémée, Geometric Galois actions 2, London Math. Soc. Lecture Note Ser. 243, Cambridge Univ. Press, Cambridge, 1997, 313-324.

[10] P. Lochak AND L. Schneps, The universal Ptolemy-Teichmüller groupoid, Geometric Galois actions 2, London Math. Soc. Lecture Note Ser. 243, Cambridge Univ. Press, Cambridge, 1997, 325-347.

[11] R. C. Penner, Universal constructions in Teichmüller theory, Adv. Math. 98 (1993), 143215.

[12] R. C. Penner, The universal Ptolemy group and its completions, Geometric Galois actions 2 , London Math. Soc. Lecture Note Ser. 243, Cambridge Univ. Press, Cambridge, 1997, 293 312 .

Graduate School of Mathematical Sciences

UNIVERSITY OF TOKYO

Komaba, Meguro

TOKYO 153-8914

JAPAN

E-mail: tsuboi@ms.u-tokyo.ac.jp 